\title{
BMJ Open Factors associated with mental health outcomes across healthcare settings in Oman during COVID-19: frontline versus non-frontline healthcare workers
}

\author{
Muna Alshekaili, ${ }^{1}$ Walid Hassan, ${ }^{1}$ Nazik Al Said, ${ }^{1}$ Fatima Al Sulaimani, ${ }^{1}$ \\ Sathish Kumar Jayapal, ${ }^{2}$ Adhra Al-Mawali, ${ }^{2}$ Moon Fai Chan, ${ }^{3}$ \\ Sangeetha Mahadevan, ${ }^{4}$ Samir Al-Adawi (i) ${ }^{4}$
}

To cite: Alshekaili M, Hassan W, Al Said N, et al. Factors associated with mental health outcomes across healthcare settings in Oman during COVID-19: frontline versus non-frontline healthcare workers. BMJ Open 2020;10:e042030. doi:10.1136/ bmjopen-2020-042030

- Prepublication history for this paper is available online. To view these files, please visit the journal online (http://dx.doi. org/10.1136/bmjopen-2020042030).

Received 23 June 2020 Revised 30 August 2020 Accepted 25 September 2020

Check for updates

(c) Author(s) (or their employer(s)) 2020. Re-use permitted under CC BY-NC. No commercial re-use. See rights and permissions. Published by BMJ.

For numbered affiliations see end of article.

\section{Correspondence to}

Dr Samir Al-Adawi;

samir.al-adawi@fulbrightmail. org

\section{ABSTRACT}

Objective This study aims to assess and compare demographic and psychological factors and sleep status of frontline healthcare workers (HCWs) in relation to nonfrontline HCWs.

Design, settings, participants and outcomes This cross-sectional study was conducted from 8 April 2020 to 17 April 2020 using an online survey across varied healthcare settings in Oman accruing 1139 HCWs.

The primary and secondary outcomes were mental health status and sociodemographic data, respectively. Mental health status was assessed using the Depression, Anxiety, and Stress Scale (DASS-21), and insomnia was evaluated by the Insomnia Severity Index. Samples were categorised into the frontline and non-frontline groups. $\chi^{2}$ and t-tests were used to compare groups by demographic data. The Mantel-Haenszel OR was used to compare groups by mental health outcomes adjusted by all sociodemographic factors.

Results This study included 1139 HCWs working in Oman. While working during the pandemic period, a total of 368 (32.3\%), 388 (34.1\%), 271 (23.8\%) and 211 $(18.5 \%)$ respondents were reported to have depression, anxiety, stress and insomnia, respectively. HCWs in the frontline group were 1.5 times more likely to report anxiety $(0 R=1.557, p=0.004)$, stress $(0 R=1.506, p=0.016)$ and insomnia $(0 R=1.586, p=0.013)$ as compared with those in the non-frontline group. No significant differences in depression status were found between the frontline and non-frontline groups $(p=0.201)$.

Conclusions To our knowledge, this is the first study to explore the differential impacts of the COVID-19 pandemic on different grades of HCWs. This study suggests that frontline HCWs are disproportionally affected compared to non-frontline HCWs, with managing sleep-wake cycles and anxiety symptoms being highly endorsed among frontline HCWs. As psychosocial interventions are likely to be constrained owing to the pandemic, mental healthcare must first be directed to frontline HCWs.

\section{INTRODUCTION}

COVID-19, a new strain among the class of coronavirus, has been reported to have first manifested in humans in December 2019,
Strengths and limitations of this study

- The study accrued 1139 participants of which 574 were working as frontline healthcare workers (HCWs) (565 non-frontline workers) serving patients with COVID-19 in different categories of healthcare settings in Oman.

- The following tools used were used alongside the collection of demographic information: The depression, Anxiety and Stress Scale (DASS-21) and Insomnia Severity Index (ISI).

- This nationally representative study is the first of its kind to investigate the differences in magnitude and the covariates of stress and distress between frontline and non-frontline HCWs in Oman.

- The use of an online survey and the use of symptom checklists (DASS, ISI), which are typically no match for the 'gold-standard' interviews.

- It is also not clear whether the observed mental health outcomes constitute adjustment disorders/ acute stress reaction or present a chronic-type and thus irreversible psychological distress.

subsequently triggering a global pandemic. Among the countries affected, specifically in the Arabian Gulf, is Oman. On 24 February 2020, Oman reported its first two cases testing positive for COVID-19. The initial report implicated the spread of COVID-19 in Oman via citizens who had travelled abroad. ${ }^{2}$ More recently, the Ministry of Health $(\mathrm{MoH})$ has reported an increasing number of people being diagnosed with COVID-19 with a few deaths and multiple recoveries. ${ }^{3}$ On 11 April, the results of mass testing by the MoH indicated approximately 500 cases per day that were confirmed to have COVID-19. This trend gradually showed only an upward trend with numbers surging up to over 1000 confirmed cases per day, indicating an increased number of cases who were becoming critically ill with some of them losing their lives. ${ }^{45}$ With the 
ever-growing number of confirmed and suspected cases, the workload of healthcare workers (HCWs) has been overwhelming. The long and irregular hours of such continuous and heavy volumes of work have the potential to trigger stress and distress.

Empirical evidence suggests that stress associated with a period of tribulation tends to weaken the immune system, further increasing the risk of diseases. ${ }^{6}$ Given this fact, in addition to having a high risk of contracting COVID-19, partly attributed to suboptimal protection, ${ }^{78} \mathrm{HCWs}$ are prone to poor mental health outcomes. ${ }^{9} 10$ Therefore, early detection among HCWs has the potential to "preempt' the development of intransigent and an advanced pathology of mental health outcomes, thereby helping to reduce the less desirable trend of having compromised HCWs during a pandemic.

The prevalence of stress and distress during times of great tribulation and seismic political, social and economic situations have been extensively investigated. ${ }^{11}$ Studies have shown a significant peak of poor coping, maladjustment and the development of emotional disorders in the wake of such unpredictable times. ${ }^{12}$ With the current global pandemic of COVID-19, Holmes et $\mathrm{al}^{13}$ have emphasised the importance of giving priority to all three tiers of social, psychological and biological health. As stress and distress have commonly been reported among HCWs, often outshining the rate observed in the general population, ${ }^{14-16}$ the question remains whether there are differences in magnitude and the covariates of stress and distress among those working on the frontlines and those who are not. This hypothesis has received scant attention.

While impressionistic reports on the psychosocial issues among HCWs have emerged in Oman, ${ }^{10}$ there is a dearth of studies that address these issues among a nationally representative sample of HCWs. This study from Oman aims to fill this gap in the existing literature. Thus, this study assessed and compared the demographic and psychological factors and sleep status of frontline HCWs versus non-frontline HCWs. Understanding demographic factors that have the potential to tamper with relevant preventative measures and knowing if their magnitude is higher among frontline HCWs will help inform the urgent mechanisms that are needed to preserve the wellbeing and resilience of such subtypes of HCWs. ${ }^{13}$

\section{METHODS}

\section{Setting and participants}

This cross-sectional study was conducted from 8 April 2020 to 17 April 2020 across varied healthcare settings in the country. Oman has a universal free healthcare system and is divided into primary, secondary, tertiary and polyclinics. ${ }^{17}$ According to the Ministry of Health $(\mathrm{MoH})$ of Oman, the first point of contact with healthcare is primary healthcare. If the service seeker requires secondary or tertiary care, they are then referred or transported to the relevant catchment areas providing secondary or tertiary care services.

With the persisting circumstances of social distancing, the study proforma was disseminated using emails of representative HCWs working in different regions of the country. ${ }^{18}$ The inclusion criteria consisted of the HCW workers designated to work in healthcare settings that dispense care for people with COVID-19. In Oman, HCWs generally work across three settings: $\mathrm{MoH}$, governmental non-MoH sector and the private sector. The present HCWs constitute only those affiliated with the $\mathrm{MoH}$. The HCWs who were quarantined/on leave or did not provide informed consent for the present study or provided incomplete responses were all excluded.

Oman has 11 administrative regions known as governorates or muhafazah. ${ }^{18}$ Concerted efforts were made to accrue participants from all such regions in the country. One relevant clinical department was randomly sampled from each chosen healthcare setting, and all HCWs in this department were asked to participate in this study. This study randomly selected one department under the $\mathrm{MoH}$ from each governorate. According to manpower statistics from the MoH in 2018, there are 39303 HCWs under the $\mathrm{MoH}$, hence around 3573 (39303/11) HCWs worked for each governorate. In each governorate, there were about nine units/departments, with about 397 HCWs (3573/9) working in each department. Hence, in total, 4367 HCWs $(397 \times 11)$ were sent the online survey and the resulting response rate stood at $1167 / 4367=26.7 \%$.

The required sample size corresponding to an acceptable margin of error for proportion (0.1) was calculated. The proportion of HCWs with psychological comorbidity was estimated at $35 \%$, based on an earlier SARS and COVID-19 outbreak report. ${ }^{9} 1920$ To allow for analysis of the relevant subgroups, the investigators of this study increased the sample size by $50 \%$ intending to reach at least 1070 participants. The study proforma was available in both Arabic and English and could be accessed via an online platform (Google document) and any information about this study was in the form itself. All respondents provided informed consent. At the end of the study survey, 1160 HCWs returned a fully completed study proforma.

\section{Outcomes and covariates}

The primary outcomes of this survey are psychological factors and self-reported sleeping problems. Psychological symptoms were collected by the Depression, Anxiety and Stress Scale (DASS-21); it is a self-report screening checklist designed to measure the negative feelings that are broadly categorised as depressive symptoms, anxiety and stress. ${ }^{21}$ Both the English and non-English (including Arabic) versions of DASS-21 have been found to have adequate internal consistency (Cronbach's alpha scores of >0.7) ${ }^{21}{ }^{22}$ DASS-21 has also been used in Oman and has been reported to have adequate Cronbach's $\alpha$ for the three subscales. The present study used the following cut-offs: depression $\geq 10$; anxiety $\geq 8$ and stress $\geq 16{ }^{22}$ We used the Insomnia Severity Index (ISI) to solicit the 
presence of subject's self-reported sleeping problems. ISI is a 7-item self-report questionnaire tapping into the severity of insomnia with each item of the scale tap into (1) 'perceived severity of difficulties initiating sleep', (2) 'difficulties staying asleep', (3) 'early morning awakenings', (4) 'satisfaction with current sleep pattern', (5) 'interference with daily functioning', (6) 'noticeability of impairment attributed to the sleep problem' and (7) 'degree of distress or concern caused by the sleep problem' ${ }^{23}$ Both English and non-English versions of the ISI (including the Arabic version) have been found to have adequate internal reliability (Cronbach's alpha scores of $>0.7) .{ }^{24}$ A 5-point Likert scale was used to rate each item (e.g, $0=$ no problem; $4=$ very severe problem), yielding a total score ranging from 0 to 28. A previous study suggested that a cut-off score of 14 was deemed adequate for detecting clinical insomnia with a sensitivity of $82.4 \%$ and specificity of $82.1 \% .^{25}$

\section{Sociodemographic factors}

The secondary outcome was the sociodemographic data (nationality, gender, age, marital status), type of medical setting (primary, secondary, tertiary care or polyclinic) and whether respondents were directly engaged in clinical activities such as diagnosing, treating or providing nursing care to patients with elevated temperatures or patients with confirmed COVID-19 infection. Those who responded as diagnosing, treating or providing nursing care were identified as 'frontline HCWs'. Those participants who had no contact with the units assigned to handle services for COVID-19 patients were defined to constitute second-line workers or 'non-frontline HCWs'. Participants' job type (physician, nurse and allied healthcare professional) was also sought. Allied healthcare professions included pharmacists and other medical staffing including laboratory technicians. Finally, the participants were also asked whether they had previously sought consultation for psychiatric disorders ('yes' /'no').

\section{Ethical issues}

This study adhered to the American Association for Public Opinion Research reporting guidelines. ${ }^{26}$ Ethical approval was obtained before the commencement of the study from the local Institutional Review Board (IRB), Directorate General of Planning and Studies, Centre of Studies and Research, MoH (MOH/DGPS/ CSR/20/2311). Written consent was sought from participants and they were told specifically that their involvement could be terminated if they wish so without undue consequences. The survey was anonymous and confidentiality of information was assured.

\section{Statistical analysis}

Data analysis was performed using SPSS statistical software V.23.0 (IBM Corp). Descriptive statistics were used to explore the profile of the samples in terms of their demographic and psychological outcomes. Samples were categorised into the frontline and non-frontline groups. $\chi^{2}$ and independent t-tests were used to compare groups by sociodemographic. Mantel-Haenszel OR was used to compare groups by psychological and self-reported sleeping problems adjusted by HCWs' job type, and other sociodemographic factors. All significant tests were set at $5 \%$ alpha level.

\section{Patient and public involvement}

It was not appropriate or possible to involve patients or the public in the design, or conduct, or reporting, or dissemination plans of our research.

\section{RESULTS}

This cross-sectional study was conducted using an online survey from 8 April 2020 to 17 April 2020 across different healthcare services in Oman. In total, we received 1167 questionnaires of which 28 were determined to be incomplete on examination. Thus, we only included 1139 records for further analysis.

\section{Demographic and psychological outcomes of the study samples}

In table 1, among the 1139 HCWs, 228 (20.0\%) are males, and $911(80.0 \%)$ are females. Their average age was $36.3 \pm 6.5($ mean $\pm \mathrm{SD})$ ranging from 21 to 65 years. The majority were Omani $(\mathrm{n}=981,86.1 \%)$ and were married $(\mathrm{n}=987,86.9 \%)$. A total of $574(50.4 \%)$ were directly involved in diagnosing, treating and taking care of confirmed or suspected cases of COVID-19 (frontline group). There were 390 (34.2\%), 164 (14.4\%), 478 $(42.0 \%)$ and $106(9.3 \%)$ working in primary healthcare, secondary healthcare, tertiary healthcare and polyclinics, respectively. Among those HCWs, 384 (33.7\%), 449 $(39.5 \%)$ and 305 (26.8) were physicians, nurses and allied health professionals, respectively. Concerning psychological outcomes, 368 (32.3\%), 388 (34.1\%), 271 (23.8\%) and $211(18.5 \%)$ respondents reported symptoms of depression, anxiety, stress and insomnia, respectively, while working during the pandemic period.

\section{Comparison of frontline and non-frontline staff on demographic and psychological factors and self-reported sleeping problems}

In table 1, significant differences were found between the two presently defined cohorts of HCWs-frontline and non-frontline groups. The frontline group comprised of members younger in age $(36.3 \pm 6.5, \mathrm{p}=0.004)$ with more of them being non-Omani $(\mathrm{n}=94,59.5 \%, \mathrm{p}=0.014)$, physicians and nurses $(\mathrm{n}=490,58.8 \%, \mathrm{p}<0.001)$, not married $(\mathrm{n}=90,60.4 \%, \mathrm{p}=0.008)$, handled COVID-19 cases $(\mathrm{n}=372,81.2 \%, \mathrm{p}<0.001)$ and working in primary healthcare setting $(\mathrm{n}=242,62.1 \%, \mathrm{p}<0.001)$ as compared with the non-frontline group. With regard to psychological outcomes, members of the frontline group were 1.5 times more likely to have anxiety $(\mathrm{OR}=1.557, \mathrm{p}=0.004)$ and stress $(\mathrm{OR}=1.506, \mathrm{p}=0.016)$ as compared with the non-frontline group. In considering self reported sleeping problems, 
Table 1 Comparison of the frontline with non-frontline staff in association of demographic and psychological factors, and self-reported sleeping problems during the impacts of COVID-19 in Oman

\begin{tabular}{|c|c|c|c|c|c|}
\hline \multirow[b]{3}{*}{ Variables } & \multirow[b]{2}{*}{ Total $(n=1139)$} & \multirow{2}{*}{$\begin{array}{l}\text { Frontline HCWs } \\
\text { Yes }(n=574)\end{array}$} & \multirow{2}{*}{$\begin{array}{l}\text { Non-frontline HCWs } \\
\text { No }(n=565)\end{array}$} & \multirow[b]{3}{*}{ Statistics* } & \multirow[b]{3}{*}{$P$ value } \\
\hline & & & & & \\
\hline & n (\%) & n (\%) & n (\%) & & \\
\hline \multicolumn{6}{|l|}{ Age (years) } \\
\hline Mean \pm SD & $36.3 \pm 6.5$ & $35.8 \pm 6.1$ & $36.9 \pm 6.8$ & $2.884 \dagger$ & 0.004 \\
\hline Female & $911(80.0)$ & $472(51.8)$ & $439(48.2)$ & & \\
\hline \multicolumn{6}{|l|}{ Nationality } \\
\hline Omani & $981(86.1)$ & $480(48.9)$ & $501(51.1)$ & 6.075 & 0.014 \\
\hline Non-Omani & $158(13.9)$ & $94(59.5)$ & $64(40.5)$ & & \\
\hline \multicolumn{6}{|c|}{$\begin{array}{l}\text { Previous sought mental health } \\
\text { consultation }\end{array}$} \\
\hline No & $1013(89.3)$ & $512(50.5)$ & $501(49.5)$ & 0.013 & 0.910 \\
\hline Yes & $122(10.7)$ & $61(50.0)$ & $61(50.0)$ & & \\
\hline \multicolumn{6}{|l|}{ Marital status } \\
\hline Not married & $149(13.1)$ & $90(60.4)$ & 59 (39.6) & 6.930 & 0.008 \\
\hline Married & $987(86.9)$ & $482(48.8)$ & $505(51.2)$ & & \\
\hline \multicolumn{6}{|c|}{$\begin{array}{l}\text { Number of COVID- } 19 \text { cases were } \\
\text { handled } \neq\end{array}$} \\
\hline Tertiary & $478(42.0)$ & $231(48.3)$ & $247(51.7)$ & & \\
\hline Polyclinic & $106(9.3)$ & $31(29.2)$ & $75(70.8)$ & & \\
\hline \multicolumn{6}{|c|}{ Psychological symptoms } \\
\hline Yes & $368(32.3)$ & $196(53.3)$ & $172(46.7)$ & $1.219 \S$ & 0.201 \\
\hline No & $771(67.7)$ & $378(49.0)$ & $393(51.0)$ & & \\
\hline Yes & $388(34.1)$ & $217(55.9)$ & $171(44.1)$ & $1.557 \S$ & 0.004 \\
\hline No & $751(65.9)$ & $357(47.5)$ & $394(52.5)$ & & \\
\hline Yes & $271(23.8)$ & $154(56.98)$ & $117(43.2)$ & $1.506 \S$ & 0.016 \\
\hline No & $868(76.2)$ & $420(48.4)$ & $448(51.6)$ & & \\
\hline \multicolumn{6}{|c|}{ Self-reported sleeping problems } \\
\hline \multicolumn{6}{|c|}{ Insomnia Severity Index } \\
\hline Yes & $211(18.5)$ & $120(56.9)$ & $91(43.1)$ & $1.586 \S$ & 0.013 \\
\hline No & $928(81.5)$ & $454(48.9)$ & $474(51.1)$ & & \\
\hline
\end{tabular}

${ }^{*} \chi^{2}$.

†t-Statistic.

†Two missing records.

§Mantel-Haenszel OR adjusted by all sociodemographic factors.

HCWs, healthcare workers. 
the frontline group was 1.5 times more likely to experience the issues of the non-frontline group $(\mathrm{OR}=1.586$, $\mathrm{p}=0.013)$. No significant differences in depression status were found between the two groups $(p=0.201)$.

\section{DISCUSSION}

Various mechanisms have been proposed to come to grip with the COVID-19 pandemic, including travel restrictions, quarantines and curfews that, in turn, have severely disrupted the social and economic activities of the society, nation or for that matter the world. ${ }^{27}$ While the impact of socioeconomic activities due to COVID-19 has been widely acknowledged in the countries of the Arabian Gulf, ${ }^{28}$ what has been overlooked is the fact that HCWs are in the frontline in the COVID-19 pandemic, which, in turn, would suggest the importance of examining their resilience in the light of those challenges.

Being a 'once-in-a-century pandemic', ${ }^{29}$ some of the misgivings affecting HCWs include the fear of contracting a lethal virus and spreading it to the rest of their social network, lack of evidence-based prevention and intervention, lack of essential protective gear and the fact that the pandemic requires protracted shifts with a high volume of patients with different degrees of pathology and severity. ${ }^{30-43}$ This would imply that HCWs are now working in a uniquely hazardous situation and are thus vulnerable to stress and distress. In addition to operational stresses, some preliminary studies have suggested that psychosocial dysfunctions are rife among HCWs. ${ }^{31} 35$ A recent systematic literature review and meta-analysis covering the literature of the prepandemic COVID-19 period suggest that $7.0 \%-75.2 \%$ of HCWs are burned out. ${ }^{36}$ This huge discrepancy in the prevalence of burnout hinges on country-specific factors, applied instruments and cut-off-criteria for burnout symptomatology. ${ }^{37}$ The prevalence of burnout among HCWs appears to outstrip the general population. ${ }^{38}$ Similarly, in addition to burnout, the prevalence of depressive symptoms, anxiety and stress among HCWs are also higher than the general population. ${ }^{14-16}$ However, since the higher level of stress and distress among HCWs as compared with the general population has been a trend existing even before the pandemic, it not clear whether the emerging high level of mental health outcomes owes its onset to COVID-19. One approach to disentangling this issue is to compare mental health outcomes between frontline HCWs versus non-frontline HCWs. This study had therefore embarked on the assessment and comparison of demographic and psychological factors and sleep status of frontline versus non-frontline HCWs.

The present study accrued 1139 HCWs from different parts of the country. As the HCWs in Oman are predominantly female ${ }^{39}$ this study is in line with the observed 'effeminisation' of healthcare as $80.0 \%$ of the present participants were female. Approximately $50 \%$ fulfil the present definition of 'frontline HCWs' who, in their clinical practices, diagnose, treat and take care of confirmed or suspected cases of COVID-19 in their respective clinics across the country. The cohort consisted of physicians, nurses and allied health professionals.

To tap into the levels of depression, anxiety and stress, the DASS-21 was used. Of the present cohort, comprised of both frontline and non-frontline HCWs, $32.3 \%$ endorsed caseness for depressive symptoms, $34.1 \%$ for anxiety and 23.8\% for stress. In Singapore, among HCWs using DASS21, Tan et $a l^{40}$ have reported $8.9 \%$ caseness for depression, $14.5 \%$ for anxiety and $6.6 \%$ for stress. Using different screening tools, Lai et at have reported $50.4 \%, 44.6 \%$ and $71.5 \%$ symptoms of depression, anxiety and distress, respectively, while Wang et $a t^{20}$ reported that $61.6 \%$ of their sample of HCWs endorsed self-reported sleep problems, $22.6 \%$ anxiety symptom and $35 \%$ depressive symptoms among HCWs in Hubei province in China. Lai's study indicated that $34.0 \%$ of their sample had an elevated score of insomnia that appears to be lower compared with the prevalence of $38.9 \%$ among HCWs investigated as part of the studies included in their systematic review and meta-analysis. ${ }^{35}$ Putting these studies together and within the background of the general population, other than the lower rate of depression in Singapore, the magnitude of mental health outcomes appears to be higher among HCWs when compared with the general population. In the general population, the prevalence of depression, anxiety and insomnia have been estimated to be $11.1 \%,{ }^{14}$ $5.3 \%, 7.3 \%{ }^{15}$ and $10 \%-30 \%,{ }^{16}$ respectively. Low mental health outcomes among HCWs in Oman and Singapore could be attributed to the preparedness phase the country underwent as the first cases were registered much later than when the WHO declared COVID-19 a global pandemic. ${ }^{1}$ While studies on the status of mental health outcomes and self-reported sleep problems have been forthcoming from different parts of the world, many of them are single-centre ${ }^{10}$ and regional studies ${ }^{9}$ with some of the catchment areas not being defined. ${ }^{40}$ A study with a nationally representative sample of HWCs taking into account both the frontline and non-frontline are therefore warranted.

The second aim of the present study was to compare demographic and psychological outcomes among frontline and non-frontline HCWs. The present data suggest that frontline HCWs are likely to be younger, single, physicians or nurses working in primary healthcare and are required to handle COVID-19 cases. The majority of frontline HCWs were non-Omani, a trend that is worth contemplating. Despite the effort to 'Omanize' the healthcare infrastructure, foreign nationals still form the bulk of HCWs in Oman. ${ }^{41}$ The COVID-19 pandemic has resulted in travel restrictions and an expected economic recession, job insecurity and being cut-off from their country of origin for the migrant population. ${ }^{42}$ It remains to be seen whether these factors have rendered nonOmani HCWs to be more vulnerable to the presently observed mental health outcomes.

In psychological outcomes, compared with nonfrontline HCWs, frontline HCWs were more likely to 
endorse anxiety symptoms and stress. A similar trend was observed with insomnia. Interestingly, the depressive symptoms did not emerge as being significant in the equation employed to differentiate between frontline versus non-frontline HCWs. Oman is characterised by a collectivistic society that is in direct contrast to western individualistic societies. ${ }^{43}$ In such a society, anxiety symptoms ('I experienced trembling in the hands') and stress ('I felt that I was using a lot of nervous energy') tend to be perceived to be a veneer of physical symptoms and are therefore likely to be endorsed. In contrast, depressive symptoms ('I felt down-hearted and blue') are thought to be more of a weakness of character than a manifestation of 'disease'. As psychological outcomes are increasingly recognised to emerge as a consequence of COVID-19, ${ }^{13}$ more studies are needed to decipher the culturally specific idioms of distress intimately tied to mental health outcomes during the pandemic.

\section{Limitations}

Most psychosocial studies of this nature tend to have many limitations owing to the amorphous variables under scrutiny. First, conducting a nationwide survey requires proper logistics, which was not feasible during the lockdown. An online survey is known to be marred by the fact that it tends to accrue a selective population who are technologically savvy and more familiar with the evolving 'internet culture'. ${ }^{44}$ Notwithstanding such a view, this study appears to have reached its targeted population based on the estimated sample size. Oman has established that $>71 \%$ of the total population (4.6 million) has access to internet services. ${ }^{44}$ Second, the DASS-21 and ISI are no match for the 'gold-standard' interviews such as those that follow the Diagnostic and Statistical Manual of Mental Disorders and WHO Composite International Diagnostic Interview. However, quick symptom checklists such as DAS-21 and ISI are the only viable tools to conduct such a study given the current circumstances. ${ }^{9}$ Related to this, future studies could employ objective measures to solicit sleep architecture. Lastly, time factors are also considered important for quantifying the presence of psychological disorders. Within this view, it is not clear whether the observed mental health outcomes constitute adjustment disorders/acute stress reaction or present a chronic-type and thus irreversible psychological distress. Follow-up studies in this regard are therefore warranted.

\section{CONCLUSION}

COVID-19, a new strain among the class of Coronavirus, has recently gripped all corners of the world triggering a global public health emergency. Within the background of high rates of poor coping among HCWs even before the pandemic, studies are needed to explore how frontline HCWs fare compared with non-frontline HCWs in this regard. This study highlighted and appeared to be congruent with other studies in suggesting that the COVID-19 outbreak has triggered a higher rate of depressive symptoms, anxiety and insomnia among HCWs. In comparing frontline and non-frontline HCWs, the present data suggested that frontline HCWs were likely to be younger non-Omani physicians or nurses who were single, and working in primary healthcare. It is therefore paramount to offer timely psychological intervention for the HCWs to promote coping and resilience among these vulnerable HCWs.

\section{Author affiliations}

${ }^{1}$ Al Masarra Hospital, Ministry of Health, Muscat, Oman

${ }^{2}$ Centre of Studies \& Research, Directorate General Planning, and studies, Ministry of Health, Muscat, Oman

${ }^{3}$ Family Medicine \& Public Health, College of Medicine and Health Sciences, Sultan Qaboos University, Muscat, Oman

${ }^{4}$ Department of Behavioral Medicine, College of Medicine and Health Sciences,

Sultan Qaboos University, Muscat, Oman

\section{Twitter Muna Alshekaili @malshekaili}

Contributors MA, WH, NAS, FA and SK had full access to all of the data in the study and take responsibility for the integrity of the data and the accuracy of the data analysis. Concept and design: MA and SA-A. Acquisition, analysis or interpretation of data: MA, WH, SK, AA-M and MFC. Drafting of the manuscript: MA, WH and SA-A. Critical revision of the manuscript for important intellectual content: SA-A, SM and MFC. Statistical analysis: WH and MFC. Administrative, technical or material support: SK and AA-M. Supervision: MA and WH.

Funding The authors have not declared a specific grant for this research from any funding agency in the public, commercial or not-for-profit sectors.

Competing interests None declared.

Patient and public involvement Patients and/or the public were not involved in the design, or conduct, or reporting, or dissemination plans of this research.

Patient consent for publication Not required.

Provenance and peer review Not commissioned; externally peer reviewed.

Data availability statement All data generated and analysed during this study are included as part of this article.

Open access This is an open access article distributed in accordance with the Creative Commons Attribution Non Commercial (CC BY-NC 4.0) license, which permits others to distribute, remix, adapt, build upon this work non-commercially, and license their derivative works on different terms, provided the original work is properly cited, appropriate credit is given, any changes made indicated, and the use is non-commercial. See: http://creativecommons.org/licenses/by-nc/4.0/.

ORCID iD

Samir Al-Adawi http://orcid.org/0000-0002-9858-5582

\section{REFERENCES}

1 Balkhair AA. COVID-19 pandemic: a new chapter in the history of infectious diseases. Oman Med J 2020;35:e123.

2 The Arabian Stories. Coronavirus: Omanis banned from leaving the country, 2020. Available: https://www.thearabianstories.com/2020/ 03/18/coronavirus-omanis-banned-from-leaving-the-country/

3 Oman Observer. Oman reports 463 new cases, total 7,257. Available: https://www.omanobserver.om/oman-reports-463-new-cases-total$7257 /$

4 Ministry of Health. Directorate General of information technology. Sultanate of Oman, 2020. Available: https://covid19.moh.gov.om/\#/ home

5 Khamis F, Al Rashidi B, Al-Zakwani I, et al. Epidemiology of COVID-19 infection in Oman: analysis of the first 1304 cases. Oman Med J 2020;35:e141.

6 de Kloet E, Joëls M. Mineralocorticoid receptors and glucocorticoid receptors in HPA stress responses during coping and adaptation. Oxford Research Encyclopedia of Neuroscience 2020.

7 Barranco R, Ventura F. Covid-19 and infection in health-care workers: an emerging problem. Med Leg J 2020;88:65-6. 
8 Jin $\mathrm{Y}-\mathrm{H}$, Huang $\mathrm{Q}$, Wang $\mathrm{Y}$-Y, et al. Perceived infection transmission routes, infection control practices, psychosocial changes, and management of COVID-19 infected healthcare workers in a tertiary acute care hospital in Wuhan: a cross-sectional survey. Mil Med Res 2020;7:24

9 Lai J, Ma S, Wang Y, et al. Factors associated with mental health outcomes among health care workers exposed to coronavirus disease 2019. JAMA Netw Open 2020;3:e203976.

10 Badahdah AM, Khamis F, Mahyijari NA. The psychological well-being of physicians during COVID-19 outbreak in Oman. Psychiatry Res 2020;289:113053.

11 Shah K, Kamrai D, Mekala $\mathrm{H}$, et al. Focus on mental health during the coronavirus (COVID-19) pandemic: applying learnings from the past outbreaks. Cureus 2020;12:e7405.

12 Wang C, Pan R, Wan X, et al. Immediate psychological responses and associated factors during the initial stage of the 2019 coronavirus disease (COVID-19) epidemic among the general population in China. Int J Environ Res Public Health 2020;17:1729.

13 Holmes EA, O'Connor RC, Perry VH, et al. Multidisciplinary research priorities for the COVID-19 pandemic: a call for action for mental health science. Lancet Psychiatry 2020;7:547-60.

14 Bromet E, Andrade LH, Hwang I, et al. Cross-National epidemiology of DSM-IV major depressive episode. BMC Med 2011;9:90.

15 Baxter AJ, Scott KM, Vos T, et al. Global prevalence of anxiety disorders: a systematic review and meta-regression. Psychol Med 2013;43:897-910.

16 Roth T. Insomnia: definition, prevalence, etiology, and consequences. J Clin Sleep Med 2007;3:S7-10.

17 Mataria A, Hajjeh R, Al-Mandhari A. Surviving or thriving in the eastern Mediterranean region: the quest for universal health coverage during conflict. Lancet 2020;395:13-15.

18 Al Riyami A, Elaty MAA, Morsi M, et al. Oman world health survey: part 1 - methodology, sociodemographic profile and epidemiology of non-communicable diseases in oman. Oman Med $J$ 2012;27:425-43.

19 Lee AM, Wong JGWS, McAlonan GM, et al. Stress and psychological distress among SARS survivors 1 year after the outbreak. Can $J$ Psychiatry 2007;52:233-40.

20 Wang W, Song W, Xia Z, et al. Sleep disturbance and psychological profiles of medical staff and non-medical staff during the early outbreak of COVID-19 in Hubei Province, China. Front Psychiatry 2020;11:733.

21 Moussa MT, Lovibond P, Laube R, et al. Psychometric properties of an Arabic version of the depression anxiety stress scales (DASS). Res Soc Work Pract 2017;27:375-86.

22 Lee J, Lee E-H, Moon SH. Systematic review of the measurement properties of the depression anxiety stress Scales-21 by applying updated COSMIN methodology. Qual Life Res 2019;28:2325-39.

23 Morin CM. Insomnia: psychological assessment and management. New York: Guilford Press, 1993.

24 Suleiman $\mathrm{KH}$, Yates BC. Translating the insomnia severity index into Arabic. J Nurs Scholarsh 2011;43:49-53.

25 Gagnon C, Bélanger L, Ivers $\mathrm{H}$, et al. Validation of the insomnia severity index in primary care. J Am Board Fam Med 2013;26:701-10.
26 American association for public opinion research (AAPOR) reporting guideline. Available: https://www.aapor.org/Publications-Media/ AAPOR-Journals/Standard-Definitions.aspx

27 Bedford J, Enria D, Giesecke J, et al. COVID-19: towards controlling of a pandemic. Lancet 2020;395:1015-8.

28 Country/Territory Report - Oman; 2020: 1-49. http://ezproxysrv.squ. edu.om:2127/login.aspx?direct=true \&db=awr\&AN=143036153\&site= ehost-live\&scope $=$ site

29 Gates B. Responding to Covid-19 - A Once-in-a-Century Pandemic? N Engl J Med 2020;382:1677-9.

30 Senni M. COVID-19 experience in Bergamo, Italy. Eur Heart $J$ 2020;41:1783-4.

31 Huang J, Liu F, Teng Z, et al. Care for the psychological status of frontline medical staff fighting against COVID-19. Clin Infect Dis 2020:ciaa385.

32 Jalili M, Niroomand M, Hadavand F, et al. Burnout among healthcare professionals during COVID-19 pandemic: a cross-sectional study. medRxiv 2020.

33 Kisely S, Warren N, McMahon L, et al. Occurrence, prevention, and management of the psychological effects of emerging virus outbreaks on healthcare workers: rapid review and meta-analysis. BMJ 2020;369:m1642.

34 Chen Q, Liang M, Li Y, et al. Mental health care for medical staff in China during the COVID-19 outbreak. Lancet Psychiatry 2020;7:e15-16.

35 Pappa S, Ntella V, Giannakas T, et al. Prevalence of depression, anxiety, and insomnia among healthcare workers during the COVID-19 pandemic: a systematic review and meta-analysis. Brain Behav Immun 2020;88:901-7.

36 Erschens R, Keifenheim KE, Herrmann-Werner A, et al. Professional burnout among medical students: systematic literature review and meta-analysis. Med Teach 2019;41:172-83.

37 Rotenstein LS, Torre M, Ramos MA, et al. Prevalence of burnout among physicians: a systematic review. JAMA 2018;320:1131-50.

38 Dyrbye LN, West CP, Satele D, et al. Burnout among U.S. medical students, residents, and early career physicians relative to the general U.S. population. Acad Med 2014;89:443-51.

39 Mohamed NA, Abdulhadi NN, Al-Maniri AA, et al. The trend of feminization of doctors' workforce in Oman: is it a phenomenon that could rouse the health system? Hum Resour Health 2018;16:19.

40 Tan BYQ, Chew NWS, Lee GKH, et al. Psychological impact of the COVID-19 pandemic on health care workers in Singapore. Ann Intern Med 2020;173:M20-1083.

41 Emerson C. Localization of the nursing workforce in the Sultanate of Oman. Case Studies in Global Health Policy Nursing 2018;28:85.

42 Liem A, Wang C, Wariyanti Y, et al. The neglected health of international migrant workers in the COVID-19 epidemic. Lancet Psychiatry 2020;7:e20.

43 Al-Adawi S. Adolescence in Oman. In: Arnett JJ, ed. International encyclopedia of adolescence: a historical and cultural survey of young people around the world. New York: Routledge, 2006: 713-828.

44 Belwal R, Al Shibli R, Belwal S. Consumer protection and electronic commerce in the Sultanate of Oman. Journal of Information, Communication and Ethics in Society 2020;ahead-of-print. 